\title{
Cultura académica y enseñanza-aprendizaje en educación superior. Revisión de literatura
}

Para citar este artículo | To cite this article Benítez-Restrepo, M. (2020). Cultura académica y enseñanza-aprendizaje en educación superior. Revisión de literatura. magis, Revista Internacional de Investigación en Educación, 13, 1-23. doi: 10.11144/Javeriana.m13.caea 


\title{
Resumen
}

La revisión de literatura realizada con 40 artículos publicados entre 2010 y 2016, sobre la cultura académica y su relación con el proceso de enseñanza aprendizaje, en diferentes universidades y países, evidencia el interés y reconocimiento a su importancia en el quehacer universitario, en coyunturas políticas, sociales, culturales presentes en su devenir. En tal sentido, constituye un aporte al campo del conocimiento que se construye a partir de los vacíos identificados en la bibliografía y la falta de consenso evidenciada entre los autores revisados. Finalmente, se define la cultura académica con una visión más amplia y trascendental.

\section{Palabras clave}

Cultura académica; educación superior; enseñanza; aprendizaje

\begin{abstract}
A literature review was conducted in 40 articles published between 2010 and 2016 that deal with academic culture and its relationship to the teaching-learning process in different colleges and countries. It evinces the interest and recognition to the importance of all this in the college endeavors, in the different political, social and cultural situations occurring throughout its becoming. In this vein, this work is a contribution to the field of knowledge built out of the gaps identified in the bibliography as well as the lack of consensus shown by the reviewed authors. Finally, the academic culture is defined under a broader and more transcendental vision.
\end{abstract}

\section{Keywords}

Scientific culture; higher education; teaching; learning 
Descripción del artículo | Article description

Este es un artículo de revisión que realicé en mis dos primeros años del Doctorado en Ciencias de Educación de la Pontificia Universidad Católica de Chile, en el marco del proyecto de investigación Influencia de las culturas académicas en la implementación de reformas curriculares en carreras acreditadas de universidades de alta calidad de Chile y Colombia.

\section{Introducción}

La presente revisión crítica de literatura se ubica en el campo de estudio de la cultura académica en educación superior. La revisión abarca un período comprendido entre 2010 y 2016; en estos años, el número de investigaciones en el campo de estudio aumentó. Los estudios en el campo cultura académica en educación superior han asumido a las instituciones como espacios culturales y articulado el vínculo sujeto-institución-disciplina (De Sierra-Neves, 2012). Al reconocer estas instituciones como espacios culturales, las investigaciones se han centrado en el análisis de las creencias, normas, valores y hábitos de las disciplinas; las características personales de los profesores y estudiantes en relación con la cultura institucional, al igual que en los procesos de aculturación (Lattuca, Terenzini, Harper \& Yin, 2010; Reason, Cox, Quaye \& Terenzini, 2010; Da Wan, Chapman, Zain, Hutcheson, Lee \& Austin, 2015; Yang, 2015).

En este contexto, la cultura desempeña un papel que es pertinente analizar, pues en su marco se inscriben las relaciones entre profesores, estudiantes y administrativos, así como los contenidos que se transmiten, transforman y crean en las instituciones de educación superior. La cultura hace referencia a las formas como los grupos de personas crean significados y comprensiones de sus propias experiencias (Austin, 1996). Además, los individuos que están en grupos crean sus "redes de significado" que incluyen sus valores compartidos. Otros autores se refieren a la cultura como "el colectivo de patrones conformados por normas, valores y creencias que guían el comportamiento de los individuos y los grupos: la cultura es un marco interpretativo para comprender eventos y acciones (Austin, 1996).

A pesar de que las investigaciones realizadas en el campo dan cuenta de elementos de la cultura (creencias, normas, valores y hábitos), al igual que algunos aspectos de los procesos de enseñanza-aprendizaje (interacciones y roles de profesor-estudiante), se encontraron libros, capítulos de libros y memorias de conferencias, relacionados con el objetivo de esta revisión 
crítica de literatura, aunque ninguno de ellos dio cuenta de una sistematización de las aproximaciones conceptuales de cultura académica y de su relación con los procesos de enseñanza-aprendizaje en educación superior (Biggs, 2004; Kreber, 2008; Stefani, 2009).

Además, se reconoce aquí la importancia de la cultura académica y los procesos de enseñanza-aprendizaje como parte de la naturaleza de las instituciones de educación superior que están en medio de los cambios sociopolíticos, culturales y sociales significativos en las últimas dos décadas. Así como la enseñanza se materializa en la docencia, se refleja en el aprendizaje y hace parte de la génesis de las universidades, también se ve influenciada por aspectos culturales de los que han sido parte los profesores y estudiantes, como otros lugares de origen y contextos marginales. Al igual que por tensiones institucionales — por ejemplo, entre la docencia y la investigación-, sin dejar de lado la normatividad institucional que determina el tiempo de dedicación a la enseñanza-aprendizaje, metodologías centradas en el profesor o estudiante, o las formas en las que se debe concebir la docencia.

En este escenario, la presente revisión crítica de literatura es un aporte al campo de estudio que se construye a partir de los vacíos identificados en la bibliografía consultada. Uno de ellos es la falta de unificación entre los autores revisados, con respecto a la concepción de cultura académica. Algunos la definen como un concepto constituido por diferentes tipos de culturas: de la disciplina, de la profesión académica, de la institución y de los sistemas de educación superior (Sánchez-Claros, 2014), y otros conformada por componentes — creencias, hábitos, valores, normas - (Szelényi \& Rhoads, 2013; Yang 2015).

Esta revisión crítica de literatura tiene implicaciones teóricas y prácticas para el campo de estudio. Con respecto a las teóricas, se evidencian diferentes relaciones entre los componentes conceptuales de la cultura académica, al igual que se identifican hallazgos que pueden aportar a futuros debates teóricos del campo de estudio, y a una mayor coherencia conceptual en próximas investigaciones. Por otro lado, las implicaciones prácticas están relacionadas con oportunidades para nuevos estudios, al reconocer vacíos y brechas en la relación entre la cultura académica y los procesos de enseñanza-aprendizaje. Esto, al considerarlos como un conjunto de elementos que interactúan de manera visible o poco visible en las instituciones de educación superior.

De acuerdo a lo anterior, el propósito de la presente revisión crítica de literatura es analizar las aproximaciones conceptuales de la cultura académica, al igual que las relaciones identificadas entre la cultura académica y los procesos de enseñanza-aprendizaje en los estudios empíricos abordados. El término cultura académica y las relaciones entre este y los procesos 
de enseñanza-aprendizaje se han convertido en conceptos y relaciones ampliamente aceptados, difundidos, utilizados y que, sin embargo, conllevan el riesgo de caer en impresiones y/o contradicciones que, al final, terminan restando potencia teórica a cada uno de ellos o a la dialéctica que se produce en su relación. A partir de lo anterior, las preguntas que guían la presente revisión son ¿cuáles son las aproximaciones conceptuales de "cultura académica" identificadas en investigaciones empíricas de educación superior? y ¿cómo se relacionan la cultura académica con los procesos de enseñanza-aprendizaje en educación superior?

El presente artículo está conformado por cuatro apartados. El primero de ellos hace referencia a antecedentes de la cultura académica y los procesos de enseñanza-aprendizaje; el segundo plantea el método utilizado para la identificación y selección de los artículos; el tercero expone los resultados de la revisión crítica de literatura y el último aborda la discusión del concepto de cultura académica y sus implicaciones en los procesos de enseñanza-aprendizaje en educación superior.

\section{Antecedentes relevantes sobre la concepción de cultura académica desde la mirada de sus autores y su relación con los procesos de enseñanza-aprendizaje}

A continuación, se presentan antecedentes de la cultura académica y los procesos de enseñanza-aprendizaje que aportan elementos para una mayor comprensión de los resultados y la discusión. Los procesos de enseñanza-aprendizaje se producen en contextos concretos que pueden llegar a afectar y determinar su desarrollo. En el caso específico de la educación superior, hay evidencias sobre su variabilidad según la institución, la ubicación geográfica y la diversidad de actores que intervienen en estos (Bentley \& Kyvik, 2012; Jiang, Di Napoli, Borg, Maunder, Fry \& Walsh, 2010; Lattuca, Terenzini, Harper \& Yin, 2010; Mathison, 2015; Reason, Cox, Quaye \& Terenzini, 2010; Da Wan, Chapman, Zain, Hutcheson, Lee \& Austin, 2015). Esto se evidencia en el desarrollo conceptual de la cultura académica desde la década de los 50.

En la década de los 50 se introdujo la discusión acerca de la cultura académica de las ciencias "duras" y las ciencias "blandas" en el marco de la revolución científica. En este sentido, la cultura académica era concebida como las diferentes formas en que los académicos de las ciencias mencionadas experimentaban la vida intelectual (Snow, 1959). En la década de los 70, se comprendía la cultura académica como una serie de visiones y maneras compartidas de enfrentar los procesos de enseñanza y las ansiedades e incertidumbres de la profesión docente en la universidad (Freedman, 1979). 
A partir de la década de los 80, la cultura académica fue concebida como un conjunto de creencias, valores, hábitos y normas que conforman cuatro tipos de cultura: de la disciplina, de la profesión académica, de la institución y de los sistemas de educación superior (Clark, 1980; Sánchez-Claros, 2014).

Al retomar los planteamientos de Mervin Freedman (1979), los retos en los procesos de enseñanza — junto con las ansiedades e incertidumbres de la profesión docente- fueron consecuencia de los cambios presentados en los sistemas de educación superior desde la década de los 70. Algunas de las transformaciones más significativas fueron la masificación y universalización de la educación superior, y llevaron a una serie de cambios sociopolíticos, culturales y sociales. Estos cambios se han evidenciado en reformas de los sistemas de educación superior asociadas con expansión, diversificación y privatización (Brunner, 2012; García-Guardilla, 2003; González-Ugalde, 2015).

Así, la masificación y la universalización han llevado a las instituciones de educación superior a diseñar e implementar políticas de control y recuperación de costos a través de aranceles de matrícula, generar cambios en los procesos de admisión, tener mayor diversidad de estudiantes, y a realizar ajustes en los planes de estudio (Bernasconi, 2010). También han llevado a aumentar la eficacia de la enseñanza (más alumnos por profesor), han tendido a separar la enseñanza de la investigación, ya que no es fácil realizar actividades de investigación con grandes números de estudiantes, y han generado una creciente incorporación de profesores adjuntos (de tiempo parcial) y de académicos con contratos a término fijo para atender las necesidades de la docencia (Bernasconi, 2010; Drew \& Klopper, 2014; Rhoads \& Hu, 2012; Van Roermund, Tromp, Scherpbier, Bottema \& Bueving, 2011; Thompson \& Clark, 2013; Torres-Olave, 2011).

En este contexto, las culturas de mayor relevancia de acuerdo con las formas en que los profesores valoran y se relacionan con la enseñanza son sus culturas disciplinares, institucionales y las asociadas al departamento académico (Austin, 1996). La cultura disciplinar es la que caracteriza a las diversas áreas de estudio y crea similitudes a través de facultades en diversos campus, mientras la cultura institucional vincula a todas las disciplinas dentro de una sola institución, y la cultura asociada al departamento académico resulta de la interacción localizada de normas y valores disciplinares e institucionales en un lugar determinado (Austin, 1996).

La cultura académica se refiere a las actitudes, creencias y valores de los académicos en relación con todos los aspectos de su trabajo (Yang 2015). En concreto, es un conjunto de elementos diferentes que se relacionan entre sí, pero que hacen parte de un solo concepto, que también está compuesto por las normas, los valores y las creencias asociadas con la vida 
laboral de los miembros del profesorado y la facultad en las instituciones de educación superior (Szelényi \& Rhoads, 2013). Finalmente, desde otros estudios, se entiende que la cultura académica se cruza de manera importante con la idea de organizaciones que tienen sus propias culturas, lo que significa que las organizaciones y las sociedades, a menudo, revelan patrones únicos de comportamiento, en consonancia con las normas, valores y actitudes comúnmente aceptados (Rhoads \& Hu, 2012).

Precisamente, las normas, valores y actitudes comúnmente aceptados también hacen parte de la enseñanza que es uno de los tres pilares en que se basa la labor general de los profesores de las instituciones de educación superior; los otros dos pilares son la investigación y la gestión. Por ejemplo, en las instituciones españolas, ninguna definición explícita da prioridad a uno de los pilares sobre los demás: los tres están integrados como uno solo. Por lo tanto, se espera que los profesores enmarquen su labor en los tres pilares (Rumyantseva \& Caboni, 2012). No obstante, en la literatura se ha identificado una tendencia que se fundamenta en la idea de que la calidad de los procesos de enseñanza-aprendizaje se basa en la participación en actividades de investigación o que "un buen profesor viene de un buen investigador" (Rumyantseva \& Caboni, 2012).

Por su parte, centrándose en la cultura de los procesos de enseñanzaaprendizaje, Juan Patricio Sánchez-Claros (2014) retoma la postura de Fernando López-Noguero (2005) quien distingue:

Entre un modelo tradicional centrado en el profesor que es quien señala los objetivos, dirige la clase y evalúa contenidos adquiridos; y un modelo participativo, en el que se valoran los aprendizajes procedimentales, el trabajo en equipo, la comunicación y la creatividad (p. 526).

En este contexto, Juan Patricio Sánchez-Claros (2014) considera:

El énfasis puesto en la diversificación metodológica, en la incentivación de la investigación, en el fomento del trabajo en grupos o por proyectos, y en el desarrollo de la autonomía de los estudiantes como protagonistas de la relación pedagógica, ha venido a subvertir en ocasiones algunos hábitos que gozaban de tradicional asentamiento entre las prácticas docentes que se venían llevando a cabo (p. 524).

La renovación de la función docente y una actitud del profesorado más como facilitador del aprendizaje que como transmisor del conocimiento son los aspectos fundamentales que han afectado a las prácticas docentes. "A pesar de estas notorias diferencias, en la práctica ambos modelos han de ser considerados como instrumentos al servicio de los objetivos 
de aprendizaje, y no como fines en sí mismos" (Sánchez-Claros, 2014, pp. 526-527). Después de haber planteado los antecedentes relevantes del campo de estudio de la cultura académica y su relación con los procesos de enseñanza-aprendizaje, se continúa con la descripción de la metodología utilizada en la revisión de literatura.

\section{Metodología}

Para la selección de los artículos, se realizó una exhaustiva búsqueda en bases de datos reconocidas por la comunidad académica, que incluyeran investigaciones de carácter interdisciplinario en educación. Esta búsqueda arrojó como bases de datos confiables: Web of Science y SCOPUS. Para este proceso se utilizaron los siguientes términos de búsqueda: "Academic Culture", "Teaching Culture", "Academic Culture AND disciplines AND Higher Education" y "Teaching Culture AND disciplines AND Higher Education".

Para la preselección de los artículos se establecieron los siguientes criterios: a) que reportaran resultados de estudios empíricos; b) que dieran cuenta de investigaciones en educación superior; c) que se hayan publicado entre 2010 y 2016; d) que establecieran relaciones con los procesos de enseñanza-aprendizaje. Se escogió este período por el aumento significativo de investigaciones en el campo de la "cultura académica" en educación superior (Mathieson, 2012; Simon, 2014; Yang, 2015).

Después de aplicar los criterios de inclusión, se revisaron títulos, resúmenes y resultados de ciento diecinueve (119) artículos obtenidos en este proceso de búsqueda. De estos, finalmente se seleccionaron cuarenta (40) artículos, que representan el 33,61\% de la búsqueda. Los artículos descartados en el proceso de selección, se excluyeron por dos razones: a) los artículos no reportaban resultados de estudios empíricos (capítulos de libros, libros y memorias de conferencias) y b) no se centraron en educación superior.

Durante la revisión de los 40 artículos, se identificaron y sistematizaron aspectos relevantes de los resultados, discusiones y conclusiones de cada investigación. Posteriormente, se reconocieron patrones que posibilitaron la construcción de categorías de análisis. Entre los aspectos reiterativos se encontraron diversas definiciones de cultura académica y de relaciones entre la cultura académica y los procesos de enseñanza-aprendizaje, lo que lleva a plantear dos categorías: 1) aproximaciones conceptuales y 2) relaciones entre la cultura académica y los procesos de enseñanza-aprendizaje.

La primera categoría hace referencia a los acuerdos y desacuerdos identificados en las definiciones y principios teóricos utilizados en los estudios empíricos abordados. En la segunda categoría que establece relaciones 
entre cultura académica y procesos de enseñanza-aprendizaje, se encontraron datos relacionados con las interacciones y roles. Estos se generan en contextos institucionales y se configuran con las características culturales de los profesores y estudiantes como actores principales de los procesos de formación universitarios.

\section{Resultados}

Una vez revisados y analizados los 40 artículos con resultados empíricos seleccionados bajo los criterios de búsqueda establecidos para esta investigación, se obtuvieron los siguientes resultados. Cuarenta artículos empíricos fueron identificados y analizados. El artículo menos reciente fue publicado en 2010 y el más reciente en 2016. La mayoría de esos artículos fueron publicados en 2011 (8) y 2012 (8), y los años de publicación menos frecuentes fueron 2010 (3) y 2016 (3). Los países con mayor representación fueron Australia (6) y Reino Unido (5), y con menor representación Holanda, Canadá, México, Chile y Brasil con un artículo respectivamente. En este contexto, las universidades australianas y chinas fueron las más representadas (5) en estudios internacionales comparados. A continuación, se presentan los resultados, a partir de las categorías 1) cultura académica: aproximaciones conceptuales y 2 ) relaciones entre la cultura académica y los procesos de enseñanza-aprendizaje.

\section{Cultura académica: aproximaciones conceptuales}

La sociología de la educación superior propone una definición que concibe la cultura académica como el conjunto de creencias, normas, hábitos y valores que hacen parte de los contextos universitarios (Clark, 1980). Los autores de algunos de los estudios consultados coinciden en mencionar estos mismos elementos, que son comunes y compartidos por quienes hacen parte de los espacios en los que se desarrollan los sistemas de educación superior (Cox, McIntosh, Reason \& Terenzini, 2011; Krause, 2014; Kustra, Doci, Gillard, Hondzel, Goff, Gabay, Meadows, Borin, Wolf, Ellis, Eiliat, Grose, Dawson \& Hughes, 2015; Szelényi \& Rhoads, 2013; Yang, 2015). Otros autores suman a los ya mencionados, otros componentes tales como patrones de comportamiento (Kustra, Doci, Gillard, Hondzel, Goff, Gabay, Meadows, Borin, Wolf, Ellis, Eiliat, Grose, Dawson \& Hughes, 2015; Rhoads \& Hu, 2012), prácticas (Bailey, 2013; Hrabowski, 2014; Thomsen, 2012), y normas (Szelényi \& Rhoads, 2013). En la revisión crítica de literatura, se constató que no hay un consenso sobre la definición de cultura académica entre los diferentes estudios empíricos revisados. 
En varios de los trabajos analizados no se habla directamente de cultura académica, si bien subrayan la importancia y la influencia de un contexto cultural general sobre los sistemas de educación superior. Algunos autores asumen el concepto de cultura institucional como el entorno físico, de valores y normas propias de la institución universitaria (Arambewela \& Hall, 2013; Glowacki-Dudka \& Treff, 2011; Henriques, Nagamine \& Nagamine, 2012; Lattuca, Terenzini, Harper \& Yin, 2010; Muldoon \& Wijeyewardene, 2012). "La cultura institucional marca las reglas internas de poder y establece las situaciones normalizadas de ejercicio profesional y de desarrollo de hábitos" (Sánchez-Claros, 2014, p. 526).

En otros estudios se evidencia una asimilación de la cultura académica con la cultura institucional en términos que se toman como sinónimos: como los entornos de aprendizaje y los valores personales que guían las prácticas y los comportamientos de profesores y estudiantes dentro de la institución universitaria (Kustra, Doci, Gillard, Hondzel, Goff, Gabay, Meadows, Borin, Wolf, Ellis, Eiliat, Grose, Dawson \& Hughes, 2015; PalomaresRuiz, 2011). Sin embargo, en otros análisis, se establece una diferenciación entre la estructura institucional vista como los valores organizacionales (instaurados por la universidad) y los elementos culturales propios de profesores y estudiantes. Esto resulta particularmente claro en los estudios de caso en los que se detallan formas de interacción de profesores y estudiantes que realizan intercambios entre instituciones y/o países, y en aquellos en los que el origen socioeconómico de los participantes se tiene en cuenta como un determinante de su desempeño académico (Bell, 2013; Mathieson, 2012; Mathison, 2015; Simon, 2014; Thompson \& Clark, 2013; Da Wan, Chapman, Zain, Hutcheson, Lee \& Austin, 2015). En estas situaciones se produce una interacción problemática entre la cultura de profesores y estudiantes (sus creencias, valores y hábitos propios, adquiridos en sus contextos particulares de socialización) y los marcos institucionales de las universidades.

Por otra parte, en algunos estudios se definen otros tipos de cultura, que se desprenden de las investigaciones realizadas, sin emplear necesariamente el concepto de cultura académica. Un ejemplo de ello es la cultura de la disciplina, que reúne "un conjunto activo de significados y prácticas simbólicas exhibidas por los miembros de un grupo" (Rostan \& Ceravalo, 2015; Wisker \& Claesson, 2013), en este caso, los miembros de determinada disciplina académica.

En palabras de Juan Patricio Sánchez-Claros (2014) "supone que cada disciplina tiene sus propias tradiciones, formas de pensamiento, métodos de investigación, orientación hacia la enseñanza, tópicos, símbolos y referencias bibliográficas que determinan el campo de estudio y las relaciones 
internas" (Sánchez-Claros, 2014, p. 526). Por esta misma línea, Alenoush Saroyan y Keith Trigwell (2015) mencionan la cultura de la profesión académica, según la cual las distintas facultades y escuelas universitarias presentan diferentes funciones, estructuras organizativas, objetivos, creencias y concepciones de la realidad, que se manifiestan en la forma de organizar el trabajo o en la interacción con los alumnos. Richard Bailey (2013) y Denis Feather (2016) se refieren a la cultura de la profesión académica como el conjunto de supuestos y prácticas que tienen sus raíces en la cultura colectiva de los departamentos, y que actúan como "teorías implícitas y prácticas recurrentes", que en muchos casos son tácitos y no se cuestionan.

Este concepto también es desarrollado por David Thompson y Alexander M. Clark (2013) en su investigación, quienes señalan que hay una resistencia de ciertos campos como la enfermería que, teniendo su propia estructura organizacional, persiste en determinar su identidad como campo de trabajo que tiende a ser profesionalizante y se resiste a ser como una disciplina académica, es decir, fundamentada en la investigación. Este último ejemplo permite reconocer la perspectiva de Sally Findlow (2012), con respecto al concepto de cultura de la profesión académica, que hace referencia a las características que definen una disciplina académica dentro de un campo del conocimiento, en este caso, la enfermería dentro del campo de las ciencias médicas. Sally Findlow (2012) menciona que la definición de académico es transitoria, pues depende de un contexto históricamente cambiante, lo que entra en conflicto con la noción de autoridad académica, que es sumamente problemática, al desplazar el foco de la calidad del trabajo académico a los discursos de profesionalización donde lo 'profesional' es tradicionalmente visto como una defensa de la autonomía de cada campo disciplinar frente a una estructura organizacional mayor.

Así, hay un acuerdo tácito entre los autores consultados, acerca de la importancia de considerar los elementos culturales, al estudiar problemas y escenarios en los que se desarrollan los sistemas de educación superior. Valores, hábitos, creencias y normas son componentes repetidamente mencionados en las definiciones encontradas. Estos generalmente guían las interacciones entre profesores y estudiantes que hacen parte de los medios universitarios, teniendo igualmente una importante influencia sobre los procesos de enseñanza-aprendizaje.

No obstante, el hecho de que estos elementos procedan de diferentes fuentes, instituciones académicas, profesores, estudiantes y organización institucional, pone de relieve la complejidad de intentar agruparlos en una sola definición. En este sentido, un mayor trabajo interdisciplinar permitiría encaminarse hacia la consolidación de definiciones mucho más completas que incluyan los variados elementos que se han puesto de manifiesto 
(normas, principios, prácticas, creencias y valores fundados en la disciplina y en la institución universitaria). Esto permitiría evaluar de un modo más integral las incidencias de la cultura académica en diferentes ámbitos, con el fin de mejorar los desempeños de las instituciones universitarias y favorecer mejores espacios de aprendizaje. A continuación se abordarán los hallazgos encontrados respecto a las relaciones entre la cultura académica y los procesos de enseñanza-aprendizaje, en los estudios consultados.

\section{Relaciones entre la cultura académica y los procesos de enseñanza-aprendizaje}

Como se presentó en la introducción, los procesos de enseñanzaaprendizaje forman parte de los sistemas de educación superior. Estos configuran una de las funciones de los profesores universitarios y permiten la reproducción y transformación del conocimiento dentro de una disciplina (Rumyantseva \& Caboni, 2012). Se encontraron definiciones referidas a procesos de enseñanza-aprendizaje en tres de los documentos que hicieron parte de la revisión (Mathieson, 2012; Sánchez-Claros, 2014; Simon, 2014). De hecho, en cada investigación se asumieron los procesos de enseñanza-aprendizaje sin hacer explícitamente una definición, sino señalando algunos rasgos importantes de los mismos en cada estudio o reflexión, según el caso.

Básicamente, se establecen los roles de los profesores y estudiantes dentro de la estructura de las instituciones universitarias y, a partir de ellos, se plantean diferentes problemáticas. Entre las más relevantes, los autores hacen referencia a discrepancias entre los elementos de una cultura académica disciplinar o institucional frente a una cultura propia de cada uno de los actores participantes, que puede provenir de su origen socioeconómico (Habel \& Whitman, 2016; Thomsen, 2012); de su proceso de formación dentro de la disciplina (Cox, McIntosh, Reason \& Terenzini, 2011; Findlow, 2012), de su país o lugar de origen (Adendorff, 2011; Rhoads \& Hu, 2012; Da Wan, Chapman, Zain, Hutcheson, Lee \& Austin, 2015), de la influencia de nuevas metodologías o pedagogías de innovación en los procesos de enseñanza-aprendizaje (Jiang, Di Napoli, Borg, Maunder, Fry \& Walsh, 2010; Simon, 2014; Szelényi \& Rhoads, 2013; Yang, 2015) y de creencias, valores y hábitos fundados en las experiencias de cada profesor y estudiante dentro de las disciplinas académicas (Bell, 2013; Mellado, 2011; Thompson \& Clark, 2013).

Una buena parte de los trabajos consultados pone de manifiesto que esta interacción (en algunos casos interferida por las diferentes formas de cultura que interactúan, y en otros, con evidentes esfuerzos para mejorarla) influye de modo determinante en los procesos de enseñanza-aprendizaje, 
lo que se profundizará en el apartado de la discusión. Algunos ejemplos de lo anterior son los planteamientos de Juan Patricio Sánchez-Claros (2014), quien afirma:

Entre todos los grupos de profesores, el de los noveles se revela como uno de los afectados de manera más intensa, viéndose enfrentados a un doble condicionante en su desempeño: por un lado, el correspondiente a la presión de integrarse en equipos y departamentos que cuentan ya con sus propias dinámicas de funcionamiento; y por otro, la atmósfera que en esos mismos departamentos ha creado la implementación de las novedades del Espacio Europeo de Educación Superior (p. 524).

sus campos y, por tanto se percibe como esencial para que los estudiantes aprendan (Lattuca, Terenzini, Harper \& Yin, 2010).

\section{La cultura académica: desde la concepción de los autores y su relación con el proceso de enseñanza-aprendizaje}

El concepto de cultura académica resulta sumamente importante para abordar y analizar diferentes aspectos de los sistemas de educación superior. Este estudio explora las diferentes aproximaciones conceptuales en investigaciones empíricas en instituciones universitarias, y cómo se relaciona con los procesos de enseñanza-aprendizaje. La mayoría de los trabajos consultados coinciden en definir la cultura académica como el conjunto de valores, hábitos y creencias que guían las prácticas de profesores y estudiantes en los contextos de educación superior. Otros estudios incorporan a estos elementos las normas y patrones de comportamiento tanto de los actores 
participantes (profesores y estudiantes) como de las instituciones universitarias en las cuales interactúan.

No obstante esta confluencia de aspectos, no hay un consenso acerca de su definición, pues en los artículos consultados se identifica una pluralidad de consideraciones conceptuales que conducen a establecer la existencia de diferentes tipos de cultura. La cultura institucional hace referencia a los marcos organizacionales e institucionales en los cuales se desarrollan los sistemas de educación superior. La cultura de la disciplina se determina a partir de aspectos académicos propios de cada campo de estudio. La cultura de la profesión académica incluye los aspectos del quehacer profesional y académico transversal a las diversas disciplinas, mientras que la cultura de los actores participantes (profesores y estudiantes) expresa características propias de los orígenes y contextos socioculturales de cada uno de ellos. Estas múltiples conceptualizaciones - y en ocasiones, matices del mismo concepto- llevan a concluir que la cultura académica tiene diferentes facetas que deben ser analizadas y cuya visión de conjunto permite formar un marco más amplio de comprensión de las dinámicas que se producen en los sistemas de educación superior, dentro de las instituciones universitarias.

La relación entre la cultura académica (expresada de las diferentes formas expuestas) y los procesos de enseñanza-aprendizaje fue el foco de varios de los estudios analizados en esta revisión crítica de literatura. Más allá de afirmar que la cultura académica tiene un efecto importante en cómo se desarrollan esos procesos, se hace necesario reconocer desde dónde se problematiza esa relación. Algunos aspectos están relacionados con los recursos personales de estudiantes y profesores, como sus lugares de origen y culturización, pasando por elementos institucionales como las demandas para los procesos de enseñanza-aprendizaje, que llevan en muchas ocasiones a tensiones entre docencia e investigación, y sin dejar de lado la normatividad institucional. Por lo anterior, resulta claro que la cultura académica tiene un efecto muy importante en cómo se desarrollan esos procesos y la forma en que afectan el desempeño tanto de los profesores como de los estudiantes. Chad Habel y Kirsty Whitman (2016) señalan en su trabajo que hay problemas de adaptación en una parte de los estudiantes, pues algunos de ellos provienen de contextos marginados y no cuentan con las habilidades necesarias para adaptarse a un entorno universitario que exige sus propias normas.

El problema mencionado es particularmente destacado por Jens Peter Thomsen (2012), quien evidencia que el sistema de educación danés típicamente pensado para la clase media, en realidad puede generar discriminaciones al exigir cierto tipo de prácticas culturales. Los resultados del trabajo de Rodney Arambewela y John Hall (2013) enfatizan la importancia crítica de 
las relaciones de la comunidad de acogida para los estudiantes internacionales y sus actitudes, que pueden ser amables, ambivalentes o antagónicas (Arambewela \& Hall, 2013). Para poder enfrentar esta situación se han hecho esfuerzos para implementar una pedagogía centrada en el estudiante, que tenga en cuenta las características de sus contextos socioculturales de proveniencia (Simon, 2014); sin embargo, esto no ha sido posible, precisamente, por la coexistencia de diferentes tipos de cultura en el ámbito universitario, que a menudo se contraponen (DeAngelo, Mason \& Winters, 2015).

De hecho, a partir de las conclusiones de muchos de los trabajos analizados, se evidencia un conflicto entre los procesos de enseñanza-aprendizaje y la investigación, como es concebida desde la cultura institucional. No obstante, esta dicotomía no es claramente planteada en la mayoría de los estudios; se discute en alguna medida, pero no recibe un tratamiento teórico suficientemente profundo. En algunos contextos universitarios, esta última es considerada más académica que la docencia (Adendorff, 2011; Bentley \& Kyvik, 2012; Krause, 2014; Kustra, Doci, Gillard, Hondzel, Goff, Gabay, Meadows, Borin, Wolf, Ellis, Eiliat, Grose, Dawson \& Hughes, 2015; Matthews, Lodge \& Bosanquet, 2014; Mellado, 2011) pues produce más beneficios económicos y reconocimiento (Drew \& Klopper, 2014), lo que desanima los intentos de innovación en las prácticas de enseñanza-aprendizaje por falta de incentivos (Smith, 2011).

Tal como lo mencionan Rui Yang (2015) y Katalin Szelényi y Robert A. Rhoads (2013), aquellos contextos donde la cultura institucional se caracteriza por normas muy rígidas y fijas (como la sociedad china y los países de la antigua Unión Soviética) es muy difícil implementar innovaciones pedagógicas, ya sea porque todavía prevalece una estrategia magistral en los procesos de enseñanza-aprendizaje (Cox, Mclntosh, Reason \& Terenzini, 2011), o porque en sociedades con culturas muy tradicionales, se percibe cierta desconfianza frente a nuevos modelos de enseñanza que desafían antiguas prácticas (Yang, 2015). En este caso, los efectos sobre los procesos de enseñanza-aprendizaje se identifican a través de metodologías y estrategias rígidas, contenidos teóricos, repetitivos, que no responden al contexto ni a las características particulares de cada estudiante. Uno de los grandes vacíos de las investigaciones analizadas hace referencia al papel de los estudiantes como participantes de los procesos de enseñanza-aprendizaje. Se asumen más en un lugar de actores pasivos y receptores, que un lugar interactivo, como productores de conocimiento y de nuevas formas de pedagogía (Sánchez-Claros, 2014).

Efectivamente, los estudiantes proceden de diferentes entornos culturales y en muchos casos tienen grandes dificultades para afrontar el mundo académico. Por ello, entre 2007 y 2017 se han venido realizando grandes 
esfuerzos para implementar innovaciones pedagógicas, basadas en el aprendizaje significativo, colaborativo, aprendizaje basado en problemas, entre otros. Estas se han implementado en universidades con estudiantes de primeras generaciones en educación superior y de diferentes lugares de origen.

En ese contexto, Chad Habel y Kirsty Whitman (2016) proponen que se implementen pedagogías de transición y se adopten enfoques que no se centren únicamente en los resultados cuantitativos, o lo que es lo mismo, que aborden formas flexibles de desarrollar estos procesos. Estos intentos indican que los profesores en general están conscientes de las necesidades de aprendizaje de los estudiantes, y de la importancia de abordar estos directamente y de un modo integrado.

Como consecuencia de muchos de estos intentos de reforma y reformas efectivas, se ha motivado la investigación en estos campos, pero según Bradley E. Cox, Kadian L. McIntosh, Robert D. Reason y Patrick T. Terenzini (2011), hay poca evidencia para indicar su adopción generalizada, y mucha resistencia a lograr mayores cambios. Pese a ello, cada vez es más importante comprender la necesidad de ir más allá de los enfoques genéricos para los procesos de enseñanza-aprendizaje, teniendo en cuenta los contextos de producción cultural de profesores y estudiantes (Mathieson, 2012). Las diferencias entre las culturas de procedencia tanto de profesores como de estudiantes tienen efectos claros en las dinámicas de los procesos de enseñanza-aprendizaje.

La valoración que los profesores hacen de su propia práctica varía de acuerdo con sus creencias, hábitos y valores, y de la filiación institucional a la universidad de la que forman parte. Por un lado, la sobrevaloración de la investigación — por oposición a la docencia - afecta el desempeño de los profesores, su distribución de tareas y en general su actitud frente a los estudiantes (Lattuca, Terenzini, Harper \& Yin, 2010; Van Roermund, Tromp, Scherpbier, Bottema \& Bueving, 2011). Se concluye de esta argumentación que se necesita una mejor articulación entre enseñanza e investigación (a través de reformas en las instituciones), y tener en cuenta los contextos de los cuales provienen y en donde interactúan los docentes y los estudiantes (Krause, 2014). Esto puede ser fácilmente perceptible cuando se realizan intercambios docentes.

La aculturación entendida como el proceso de adaptación de los profesores a un nuevo espacio, no solo institucional sino cultural (otra región u otro país), puede conducir a mejoras en las prácticas de enseñanza-aprendizaje, o puede dejar en claro las dificultades para asumir nuevas normas y con ello, identificarse con una cultura institucional. Por otro lado, algunos profesores exponen que las mayores fuentes de satisfacción en su trabajo son la docencia, la investigación y la naturaleza flexible del trabajo 
académico. Así, dedicar tiempo a la docencia y a la innovación en sus prácticas de enseñanza-aprendizaje es visto por ellos como una forma de nutrir su desempeño profesional, complementar su actividad como investigadores y sentirse mayormente identificados con su propia disciplina.

Entre las fuentes de frustración de los académicos se mencionan las políticas de la universidad y la burocracia, las cargas administrativas y la escasez de recursos. De cierta forma, estas entorpecen el trabajo académico y afectan los procesos de enseñanza-aprendizaje (Da Wan, Chapman, Zain, Hutcheson, Lee \& Austin, 2015). Nuevamente, es posible visualizar un conflicto entre la cultura institucional, y la cultura profesional o disciplinar de los docentes y la forma como desde ambos lados se comprenden los procesos de enseñanza-aprendizaje.

\section{Conclusiones}

La revisión crítica de literatura evidenció multiplicidad de conceptualizaciones de la cultura académica, lo que exigió un fuerte trabajo de comprensión y síntesis. Algunos artículos elaboraron su argumentación desde una concepción tácita de lo que es la cultura académica, sin proporcionar definiciones más claras o puntuales. De la misma manera, pocos artículos (3) presentaron una definición de lo que se entendía por los procesos de enseñanza-aprendizaje, mostraron de entrada sus resultados y con ello, pasaron por alto importantes elementos para una discusión, como el papel de los estudiantes en esos procesos y la importancia de las reformas en los sistemas de educación superior. En aquellos trabajos que desarrollaron estudios de caso muy puntuales, los elementos teóricos no estaban ampliamente abordados, por lo que fue necesario acudir a fuentes secundarias para poder comprender el contexto teórico en que se plantearon.

Las limitaciones planteadas son oportunidades para futuras investigaciones que se realicen en el campo de estudio. Una de ellas hace referencia al desarrollo teórico del concepto de cultura académica desde una construcción interdisciplinar que posibilite la comprensión de la complejidad de la cultura en sí misma y mucho más al ubicarla en un contexto acadé-

mico en el que se enfrentan arduos retos y desafíos cada vez más frecuentes. Por otro lado, la relación entre la cultura académica y los procesos de enseñanza-aprendizaje se convierte en un objeto de investigación poco explorado, que puede aportar a la calidad de los procesos de formación profesional de las nuevas generaciones, de las cuales depende en gran medida el desarrollo sociopolítico, económico y educativo de las sociedades contemporáneas. 
De todo lo anterior, se concluye que resulta relevante continuar por esta vía de investigación y persistir en la búsqueda de integración en la definición de cultura académica, que permita tener una visión más completa de toda la problemática asociada a los procesos de enseñanza-aprendizaje. Hasta aquí se ha visto una perspectiva fragmentada y dividida, con diferentes concepciones sobre cultura; si bien es necesario reconocer sus dinámicas propias y sus características particulares, también es indispensable generar una consideración conceptual de fondo que lleve a plantear soluciones mucho más aplicables a los diferentes contextos universitarios.

En estos contextos universitarios, se requiere una definición de cultura mucho más aplicable, que reconozca el conflicto, que permita evidenciar las disputas, los movimientos y los cambios constantes a los que se enfrentan cotidianamente los académicos, estudiantes y administrativos. Esto se propone una vez se observa en las diferentes posturas teóricas, que el término cultura académica se asume como conjunto de acuerdos que determinan las construcciones de procesos de identidad, hábitos, normas y creencias en los actores docentes que hacen vida en la universidad, sin soslayar los elementos divergentes que emergen de la cotidianidad y que coexisten con los acuerdos asumidos como patrones de comportamiento.

A partir de lo anterior, la cultura académica se define como las creencias, hábitos, normas, valores y procesos identitarios que se construyen a partir de consensos, al igual que desde desacuerdos y disputas derivadas de la presencia de percepciones y valores distintos, que hacen posible el orden cultural. Lo anterior posibilita que los sujetos en contextos disciplinares e institucionales definan quiénes son, qué hacen y por qué lo hacen. En este contexto, la cultura académica tiene efectos sobre los modos en que se produce, discute o negocia, y disemina el conocimiento (Araujo, 2015).

\section{Agradecimientos y aclaraciones}

El artículo es uno de los productos de los primeros dos años de la revisión crítica de literatura de mi tesis doctoral, que es financiada por la Comisión Nacional de Investigación Científica y Tecnológica, CONICYT, Chile.

\section{Sobre la autora}

Milena Benítez-Restrepo es candidata al Doctorado en Ciencias de la Educación de la Pontificia Universidad Católica de Chile, Chile. Magíster en Educación de la Universidad de los Andes, Colombia. Docente universitaria, ha investigado sobre enseñanza, cultura académica y currículo en educación superior. 


\section{Referencias}

Adendorff, H. (2011). Strangers in a Strange Land - On Becoming Scholars of Teaching. London Review of Education, 9 (3), 305-315. DOI: https://doi.org/10. 1080/14748460.2011.616323. Disponible en: https://www.ingentaconnect. com/content/ioep/clre/2011/00000009/00000003/art00004?crawler=true

Arambewela, R. \& Hall, J. (2013). The Interactional Effects of the Internal and External University Environment, and the Influence of Personal Values, on Satisfaction among International Postgraduate Students. Studies in Higher Education, 38 (7), 972-988. https://doi.org/10.1080/03075079.2011.615916

Araujo, K. (2015). Culturas académicas: entre reinvención y contrabando. Papeles del CEIC [Centro de Estudios sobre la Identidad Colectiva, Universidad del País Vasco], 1 (118), 1-17. http://dx.doi.org/10.1387/pceic.13014. Disponible en: https://www.ehu.eus/ojs/index.php/papelesCEIC/article/ view/13014/12457

Austin, A. E. (1996). Institutional and Departmental Cultures: The Relationship between Teaching and Research. New Directions for Institutional Research, 90, 57-66. https://doi.org/10.1002/ir.37019969009

Bailey, R. (2013). Exploring the Engagement of Lecturers with Learning and Teaching Agendas through a Focus on their Beliefs about, and Experience with, Student Support. Studies in Higher Education, 38 (1), 143-155. https://doi. org/10.1080/03075079.2011.578246

Bell, E. (2013). Organisational Culture and Learning: A Case Study. Nurse Education Today, 33 (11), 1337-1341. https://doi.org/10.1016/j.nedt.2013.02.009

Bentley, P. J. \& Kyvik, S. (2012). Academic Work from a Comparative Perspective: a Survey of Faculty Working Time across 13 Countries. Higher Education, 63 (4), 529-547. DOI 10.1007/s10734-011-9457-4

Bernasconi, A. (2010). La apoteosis del investigador y la institucionalización de la profesión académica en Chile. Estudios sobre Educación, 19, 139-163. Disponible en https://www.unav.edu/publicaciones/revistas/index.php/ estudios-sobre-educacion/article/view/4584/3952

Biggs, J. (2004). Calidad del aprendizaje universitario, Vol. 7. Madrid: Narcea Ediciones.

Brunner, J. J. (2012). La idea de universidad en tiempos de masificación. Revista Iberoamericana de Educación Superior, RIES, 3 (7), 130-145. DOI: 10.22201/iisue.20072872e.2012.7.69. Disponible en http://www.redalyc. org/pdf/2991/299129031008.pdf

Clark, B. R. (1980). Academic Culture. Higher Education Research Group, Institution for Social and Policy Studies, Yale University, Working Paper. Disponible en: https://files.eric.ed.gov/fulltext/ED187186.pdf

Cox, B. E.; Mclntosh, K. L.; Reason, R. D. \& Terenzini, P. T. (2011). A Culture of Teaching: Policy, Perception, and Practice in Higher Education. Research in Higher Education, 52 (8), 808-829. DOI 10.1007/s11162-011-9223-6.

DeAngelo, L.; Mason, J. \& Winters, D. (2015). Faculty Engagement in Mentoring Undergraduate Students: How Institutional Environments Regulate and Promote Extra-Role Behavior. Innovative Higher Education, 41 (4), 317-332. DOI 10.1007/s10755-015-9350-7 
Drew, S. \& Klopper, C. (2014). Evaluating Faculty Pedagogic Practices to Inform Strategic Academic Professional Development: A Case of Cases. Higher Education, 67 (3), 349-367. DOI: 10.1007/s10734-013-9657-1

Feather, D. (2016). Defining Academic-Real or Imagined. Studies in Higher Education, 41 (1), 110-123. DOI: 10.1080/03075079.2014.914921

Findlow, S. (2012). Higher Education Change and Professional-Academic Identity in Newly 'Academic' Disciplines: The Case of Nurse Education. Higher Education, 63 (1), 117-133. DOI 10.1007/s10734-011-9449-4.

Freedman, M. (1979). Academic Culture and Faculty Development. Orinda, California: Montaigne Publishing.

García-Guadilla, C. (2003). Balance de la década de los '90 y reflexiones sobre las nuevas fuerzas de cambio en la educación superior. En Marcela Mollis (comp.). Las universidades en América Latina: ¿reformadas o alteradas? La cosmética del poder financiero, 17-37. Buenos Aires: Consejo Latinoamericano de Ciencias Sociales, CLACSO. Disponible en: http://biblioteca.clacso. edu.ar/clacso/gt/20101109010429/mollis.pdf, http://biblioteca.clacso.edu. ar/gsdl/collect/clacso/index/assoc/D3056.dir/2gguadilla.pdf

Glowacki-Dudka, M. \& Treff, M. (2011). Forming an Institutional Culture with Multinational Administrators and Teachers at Effat College, Saudi Arabia. Intercultural Education, 22 (2), 207-222. DOI: 10.1080/14675986.20 11.567078

González-Ugalde, C. (2015). Perspectivas y desafíos de la docencia en educación superior chilena. En A. Bernasconi (ed.). La educación superior de Chile. Transformación, desarrollo y crisis, 371-407. Santiago de Chile: Centro de Estudios de Políticas y Prácticas en Educación, CEPPE, Ediciones Pontificia Universidad Católica de Chile, Ediciones UC.

Habel, C. \& Whitman, K. (2016). Opening Spaces of Academic Culture: Doors of Perception; Heaven and Hell. Higher Education Research \& Development, 35 (1), 71-83. DOI: 10.1080/07294360.2015.1133570

Henriques, A.; Nagamine, A. \& Nagamine, C. M. L. (2012). Reflexões sobre análise institucional: o caso do ensino e aprendizagem de integrais múltiplas. Bolema: Boletim de Educação Matemática, 26 (44), 1261-1288. DOI: 10.1590/S0103-636X2012000400008. Disponible en: http://www.periodi cos.rc.biblioteca.unesp.br/index.php/bolema/issue/view/939

Hrabowski III, F. A. (2014). Institutional Change in Higher Education: Innovation and Collaboration. Peabody Journal of Education, 89 (3), 291-304. DOI: 10.1080/0161956X.2014.913440

Jiang, X.; Napoli, R. di; Borg, M.; Maunder, R.; Fry, H. \& Walsh, E. (2010). Becoming and Being an Academic: the Perspectives of Chinese Staff in Two Research-Intensive UK Universities. Studies in Higher Education, 35 (2), 155 170. DOI: 10.1080/03075070902995213

Krause, K. L. D. (2014). Challenging Perspectives on Learning and Teaching in the Disciplines: the Academic Voice. Studies in Higher Education, 39 (1), 2-19. https://doi.org/10.1080/03075079.2012.690730

Kreber, C. (ed.). (2008). The University and its Disciplines. Teaching and Learning within and beyond Disciplinary Boundaries. New York: Taylor \& Francis.

Kustra, E.; Doci, F.; Gillard, K.; Hondzel, C. D.; Goff, L.; Gabay, D.; Meadows, K.; Borin, P.; Wolf, P.; Ellis, D.; Eiliat, H.; Grose, J.; Dawson, D. \& Hughes, S. 
(2015). Teaching Culture Perception: Documenting and Transforming Institutional Teaching Cultures. Collected Essays on Learning and Teaching, 8, 231-244. DOI: https://doi.org/10.22329/celt.v8i0.4267. Disponible en: https://celt.uwindsor.ca/index.php/CELT/article/view/4267

Lattuca, L. R.; Terenzini, P. T.; Harper, B. J. \& Yin, A. C. (2010). Academic Environments in Detail: Holland's Theory at the Subdiscipline Level. Research in Higher Education, 51 (1), 21-39. DOI 10.1007/s11162-009-9144-9

Mathieson, S. (2012). Disciplinary Cultures of Teaching and Learning as Socially Situated Practice: Rethinking the Space between Social Constructivism and Epistemological Essentialism from the South African Experience. Higher Education, 63 (5), 549-564. DOI 10.1007/s10734-011-9458-3

Mathison, K. (2015). Effects of the Performance Management Context on Australian Academics' Engagement with the Scholarship of Teaching and Learning: A Pilot Study. The Australian Educational Researcher, 42 (1), 97-116. DOI: 10.1007/s13384-014-0154-z

Matthews, K. E.; Lodge, J. M. \& Bosanquet, A. (2014). Early Career Academic Perceptions, Attitudes and Professional Development Activities: Questioning the Teaching and Research Gap to Further Academic Development. International Journal for Academic Development, 19 (2), 112-124. DOI: 10.1080/1360144X.2012.724421

Mellado, C. (2011). Examining Professional and Academic Culture in Chilean Journalism and Mass Communication Education. Journalism Studies, 12 (3), 375391. DOI: 10.1080/1461670X.2010.507373

Muldoon, R. \& Wijeyewardene, I. (2012). Two Approaches to Mentoring Students into Academic Practice at University. Journal of the Australian \& New Zealand Student Services Association, 39, 21-31.

Palomares-Ruiz, A. (2011). El modelo docente universitario y el uso de nuevas metodologías en la enseñanza, aprendizaje y evaluación [The Educational Model at University and the Use of New Methodologies for Teaching, Learning and Assessment]. Revista de Educación, 355, 591-604. DOI: 104438/1988-592X-RE-2011-355-038. Disponible en: http://www.educacionyfp.gob.es/dam/jcr:6a501328-72b6-4138-a12e-f65b7b5d0bc8/re35525.pdf

Reason, R. D.; Cox, B. E.; Quaye, B. R. L. \& Terenzini, P. T. (2010). Faculty and Institutional Factors that Promote Student Encounters with Difference in First-Year Courses. The Review of Higher Education, 33 (3), 391-414. DOI: 10.1353/rhe.0.0137

Rhoads, R. A. \& Hu, J. (2012). The Internationalization of Faculty Life in China. Asia Pacific Journal of Education, 32 (3), 351-365. DOI: 10.1080/02188791. 2012.711293. Disponible en: https://escholarship.org/content/qt8fd261rh/ qt8fd261rh.pdf

Roermund, T. C. van; Tromp, F.; Scherpbier, A. J.; Bottema, B. J. \& Bueving, H. J. (2011). Teachers' Ideas versus Experts' Descriptions of 'the Good Teacher' in Postgraduate Medical Education: Implications for Implementation. A Qualitative Study. BMC Medical Education, 11 (1), 1-8. DOI 10.1186/14726920-11-42

Rostan, M. \& Ceravolo, F. A. (2015). The Internationalisation of the Academy: Convergence and Divergence across Disciplines. European Review, 23 (S1), S38-S54. DOI: 10.1017/S1062798714000763 
Rumyantseva, N. L. \& Caboni, T. C. (2012). Are Academics in Kazakhstan Capable of Self-Regulation? A Study of Faculty's Normative Structure in the Midst of Higher Education Decentralization Reforms. Tertiary Education and Management, 18 (1), 1-16. DOI: 10.1080/13583883.2011.597057

Sánchez-Claros, J. P. (2014). Influencia de la cultura académica de origen en el desempeño docente de profesores universitarios noveles. Historia y Comunicación Social, 9, 523-533. Disponible en: https://revistas.ucm.es/index. php/HICS/article/download/45046/42417

Saroyan, A. \& Trigwell, K. (2015). Higher Education Teachers' Professional Learning: Process and Outcome. Studies in Educational Evaluation, 46, 92-101. DOI: 10.1016/j.stueduc.2015.03.008

Sierra-Neves, M. T. de (2012). El desarrollo de la cultura académica en instituciones de educación superior públicas en México: UNAM y UAM. En B. Y. García \& B. I. Ortiz-Molina (comps.). Reflexiones acerca del trabajo académico en el contexto iberoamericano, 63-92. Bogotá: Universidad Distrital Francisco José de Caldas. Disponible en: https://www.researchgate.net/ profile/Barbara_Sanchez3/publication/277229606_El_trabajo_academi co_del_profesor_universitario/links/5591725c08ae15962d8e2141/El-traba jo-academico-del-profesor-universitario.pdf

Simon, E. (2014). Teaching Political Science Research Methods in Hungary: Transferring Student-Centered Teaching Practices into a Subject-Focused Academic Culture. European Political Science, 13, 78-95. DOl:10.1057/eps.2013.43

Smith, K. (2011). Cultivating Innovative Learning and Teaching Cultures: A Question of Garden Design. Teaching in Higher Education, 16 (4), 427-438. DOI: 10.1080/13562517.2011.560374

Snow, C. P. (1959). The Two Cultures and the Scientific Revolution. Cambridge, New York: Cambridge University Press.

Stefani, L. (2009). Planning Teaching and Learning, Curriculum Design and Development. En H. Fry, S. Ketteridge \& S. Marshall (eds.). A Handbook for Teaching and Learning in Higher Education. Enhancing Academic Practice, Chapter 4, 40-57. New York: Routledge. Disponible en: http://biblioteca.ucv. cl/site/colecciones/manuales_u/A\%20Handbook\%20for\%20Teaching\%20 and\%20Learning\%20in\%20Higher\%20Education\%20Enhancing\%20aca demic\%20and\%20Practice.pdf

Szelényi, K. \& Rhoads, R. A. (2013). Academic Culture and Citizenship in Transitional Societies: Case Studies from China and Hungary. Higher Education, 66 (4), 425-438. DOI 10.1007/s10734-013-9614-z

Thompson, D. R. \& Clark, A. M. (2013). Academic Culture in Nursing: Devalued, Defiant or Dead? International Journal of Nursing Studies, 50 (11), 14311433. DOI:10.1016/j.ijnurstu.2013.05.009

Thomsen, J. P. (2012). Exploring the Heterogeneity of Class in Higher Education: Social and Cultural Differentiation in Danish University Programmes. British Journal of Sociology of Education, 33 (4), 565-585. DOI 10.1080/01425692.2012.659458

Torres-Olave, B. M. (2011). "There Is a World Out There": Spatial Imagination, Agency, and Academic Culture in a Mexican University Program. Comparative Education Review, 55 (1), 030-055. DOI: 10.1086/656734 
Wan, C. da; Chapman, D. W.; Zain, A. N. M.; Hutcheson, S.; Lee, M. \& Austin, A. E. (2015). Academic Culture in Malaysia: Sources of Satisfaction and Frustration. Asia Pacific Education Review, 16 (4), 517-526. DOI 10.1007/ s12564-015-9398-1

Wisker, G. \& Claesson, S. (2013). The Impact of Cross-Disciplinary Culture on Student-Supervisor Perceptions. International Journal of Doctoral Studies, 8, 21-37. Disponible en: http://www.informingscience.com/ijds/Volume8/ IJDSv8p021-037Wisker0389.pdf

Woolley, N. N. \& Jarvis, Y. (2007). Situated Cognition and Cognitive Apprenticeship: A Model for Teaching and Learning Clinical Skills in a Technologically Rich and Authentic Learning Environment. Nurse Education Today, 27 (1), 73-79. DOI:10.1016/j.nedt.2006.02.010

Yang, R. (2015). Reassessing China's Higher Education Development: A Focus on Academic Culture. Asia Pacific Education Review, 16 (4), 527-535. DOI 10.1007/s12564-015-9397-2 